\title{
Rythme d'apparition des racines primaires du maïs (Zea mays L.). III. Variations observées au champ
}

Marie-Odile JORDAN, Philippe GIRARDIN, Claude VARLET-GRANCHER*, Didier PICARD** \& Roland TRENDEL

I.N.R.A., Station d'Agronomie, B.P. 507, F 68021 Colmar

* I.N.R.A., Station de Bioclimatologie, Centre de Recherches de Poitou-Charentes, F 86600 Lusignan

La variabilité du nombre de racines primaires par entre-nœud ou groupe d'entre-nœuds induite par les facteurs du milieu a été caractérisée à partir d'un dispositif expérimental multilocal et d'une étude à partir de semis échelonnés. Des différences significatives ont été mises en évidence, tant au niveau des nombres moyens de racines qu'au niveau de la forme des distributions racinaires. La variabilité augmente avec le rang de l'entre-nœud considéré. Le nombre de racines à niveau donné dépend peu de l'émission aux entre-nœuds inférieurs. De plus, il n'y a pas de relation chez les plantes matures entre le nombre total de feuilles et le nombre total de racines. L'échelonnement de la date de semis ne modifie pas la cinétique de l'émission racinaire mais a une influence sur le nombre de racines portées par les derniers entre-nœuds.

Mots clés additionnels : Phyllochrone, somme de température, entre-nœeud.

Rate of appearance of primary roots in maize. III. Variation observed in the field.

The influence of climatic or cultural factors on the variability of primary root number was investigated. This variability was measured at several locations and in a particular experiment with staggered sowing dates. Treatments significantly affected the mean number of roots and the distribution of the roots on the internodes. The total variability increased with internode level. Root number depended slightly on the number of roots of the lower internodes. In mature plants, there was no relationship between total leaf and total root number. The staggering of sowing dates did not influence the kinetics of primary root emission, but influenced the number of roots of the last internodes.

Additional key words : Phyllochron, internode, heat units.

\section{INTRODUCTION}

Dans un premier article, nous avons étudié les rythmes d'émission des racines primaires du maïs pour une variété, «Dea », en un lieu donné (PICARD et al., 1985). Mais ces résultats ne peuvent être utilisés avec profit pour la modélisation de la croissance et du développement du maïs, que s'ils sont complétés par une étude de la variabilité liée à diverses conditions de milieu.

L'objet de ce travail est de caractériser les écarts observés dans la mise en place du système racinaire en conditions habituelles de culture. Au cas où une varia-

** Adresse actuelle: I.N.R.A., Département d'Agronomie, Centre de Recherches de Paris-Grignon, 78850 Thiverval-Grignon. bilité importante serait observée, un dispositif adéquat permettant de l'expliquer pourrait être envisagé ultérieurement. On essaiera cependant, à ce stade de l'étude, de mettre en évidence l'existence de régulations internes à la plante (phénomènes de compensation, équilibre parties aériennes-parties racinaires) dans l'installation du système racinaire.

Pour explorer une partie de la variabilité liée aux interactions climat $\times$ techniques culturales, des observations ont été effectuées sur 6 sites d'expérimentation en Alsace pendant 2 années consécutives. Celles-ci ont été complétées par une étude conduite pendant un an à Estrées-Mons (Somme) appelé Mons par la suite.

D'autre part, l'influence de la date de semis a été étudiée à Guelph (Ontario, Canada) sur un hybride denté «P 3851 » dont les caractéristiques de précocité 
sont proches de celles de «Dea ». Pour comparer les résultats obtenus sur des parcelles semées à différentes dates, on a utilisé comme norme de temps les sommes de température. En effet, parmi de nombreux auteurs, TOLLENAAR et al. (1979), TOLLENAAR \& HUNTER (1983), WARRINGTON \& KANEMASU $(1983 a, b$ et $c$ ), DUBURCQ et al. (1983), RUSSELL \& STUBER (1983), RUSSELlE et al. (1984) ont montré que la température était le facteur d'action prépondérant des mécanismes de développement.

\section{DISPOSITIF EXPÉRIMENTAL}

En Alsace, les expérimentations ont été conduites selon les normes de la grande culture pendant 2 années consécutives, 1983 et 1984, sur 6 sites (tabl. 1). Quatre sous-parcelles ont été implantées dans chaque cas, sauf pour Colmar 1 où ce nombre a été de 5 . A Mons, un dispositif à 4 répétitions a été retenu pour des observations conduites la seule année 1983. A Guelph enfin, l'essai comprenait, en 1984, 3 dates de semis (17 mai, 14 et 22 juin) avec 4 répétitions.

En Alsace, les sites ont été implantés de façon à inclure une certaine variabilité climatique. En effet, la pluviométrie augmente régulièrement du centre ( $\mathrm{Col}-$ mar et Gundolsheim), au sud (Altkirch et Flaxlanden) de cette région, alors que les températures sont beau- coup plus stables (tabl. 1a). Par rapport à la normale établie à partir des données de 1885 à 1982 à Colmar (363 mm de pluie entre avril et octobre), l'année 1983 est pluvieuse $(460 \mathrm{~mm}$ de pluie pendant la même période) alors que 1984 se rapproche de la normale avec $347 \mathrm{~mm}$ de pluie. Les températures moyennes mensuelles sont inférieures à la normale sauf en ce qui concerne les mois de juillet, août et septembre 1983. Le climat de Mons, de type océanique, est généralement plus humide et plus frais qu'en Alsace. En 1983 cependant, la pluviométrie a été beaucoup plus importante dans l'est de la France qu'à Mons. A Guelph enfin, on a un climat de type continental.

En Alsace et à Mons, les sols des stations, formés sur loess, (tabl. 1b) présentent tous une texture de limon argileux avec une teneur en matière organique faible à modérée, un $\mathrm{pH}$ alcalin ou neutre. A Guelph, la texture est de limon argilo-sableux. Compte tenu de la profondeur des sols, l'alimentation en eau des plantes des sites expérimentaux a toujours été suffisante.

Les techniques culturales ont été laissées à l'initiative des agriculteurs de façon à disposer d'une gamme de variation d'états du milieu et du peuplement comparable à celle que l'on peut observer classiquement. Ainsi les densités du maïs varient entre 6,2 et 9,5 pieds $/ \mathrm{m}^{2}$. Les états du profil (relevés au stade 7-8 feuilles) sont caractérisés par :

- une croûte de battance généralisée,

- un horizon labouré où, sur certains sites (Gun-

TABLEAU 1

Caractéristiques du milieu pédo-climatique.

Pedo-climatic caracteristics.

a) Données climatiques

Climatic data

\begin{tabular}{|c|c|c|c|c|c|c|c|c|c|c|c|c|}
\hline & & & \multicolumn{2}{|c|}{ Colmar } & \multirow{2}{*}{$\begin{array}{l}\text { Gundols- } \\
\text { heim }\end{array}$} & \multirow{2}{*}{$\begin{array}{l}\text { Flax- } \\
\text { landen }\end{array}$} & \multicolumn{2}{|c|}{ Altkirch } & \multirow{2}{*}{ Mons } & \multicolumn{3}{|c|}{ Guelph } \\
\hline & & & 1 & 2 & & & 1 & 2 & & date 1 & date 2 & date 3 \\
\hline \multirow{4}{*}{$\begin{array}{l}\text { Somme des } \\
\text { températures } \\
\text { en base } 6{ }^{\circ} \mathrm{C}\end{array}$} & Semis floraison femelle & $\begin{array}{l}1983 \\
1984\end{array}$ & $\begin{array}{l}830 \\
866\end{array}$ & $\begin{array}{l}830 \\
980\end{array}$ & $\begin{array}{l}930 \\
956\end{array}$ & $\begin{array}{r}943 \\
1080\end{array}$ & $\begin{array}{l}885 \\
820\end{array}$ & $\begin{array}{l}867 \\
790\end{array}$ & $\begin{array}{l}922 \\
-\end{array}$ & $\overline{743}$ & $\begin{array}{c}-- \\
794\end{array}$ & $\overline{816}$ \\
\hline & \multirow[t]{2}{*}{ Semis récolte } & \multirow{2}{*}{$\begin{array}{l}1983 \\
1984\end{array}$} & \multirow{2}{*}{$\begin{array}{l}1692 \\
1612\end{array}$} & \multirow{2}{*}{$\begin{array}{l}1692 \\
1655\end{array}$} & \multirow{2}{*}{$\begin{array}{l}1827 \\
1742\end{array}$} & \multirow{2}{*}{$\begin{array}{l}1884 \\
1819\end{array}$} & \multirow{2}{*}{$\begin{array}{l}1792 \\
1467\end{array}$} & \multirow{2}{*}{$\begin{array}{l}1775 \\
1447\end{array}$} & \multirow{2}{*}{$\begin{array}{l}1667 \\
-\end{array}$} & \multirow{2}{*}{$1 \overline{460}$} & - & - \\
\hline & & & & & & & & & & & \multicolumn{2}{|c|}{ non récolté } \\
\hline & $\begin{array}{l}\text { Précipitations en } \mathrm{mm} \\
\text { du 1.IV au 10.IX }\end{array}$ & $\begin{array}{l}1983 \\
1984\end{array}$ & \multicolumn{2}{|c|}{$\begin{array}{l}435 \\
316\end{array}$} & $\begin{array}{l}474 \\
317\end{array}$ & $\begin{array}{l}578 \\
335\end{array}$ & \multicolumn{2}{|c|}{$\begin{array}{l}623 \\
411\end{array}$} & 206 & \multicolumn{3}{|c|}{408} \\
\hline
\end{tabular}

b) Caractéristiques physiques des horizons labourés des parcelles expérimentales.

Physic caracteristics of the experimental plots.

\begin{tabular}{|c|c|c|c|c|c|c|c|}
\hline & \multicolumn{6}{|c|}{ Analyse physique $\%$} & \multirow{2}{*}{$\mathrm{pH}$} \\
\hline & Argile & Limon fin & Limon grossier & Sable fin & Sable grossier & Calcaire total & \\
\hline Alsace $\left(48,8^{\circ} \mathrm{N}-193 \mathrm{~m}\right)$ & $21,5 \quad(2,4)$ & $32,0 \quad(3,9)$ & $40,9 \quad(3,1)$ & $3,7 \quad(2,9)$ & $1,9 \quad(1,5)$ & $7,5 \quad(8.1)$ & $7.7 \quad(0,7)$ \\
\hline Mons $\left(50^{\circ} \mathrm{N}-85 \mathrm{~m}\right)$ & 17,4 & 21,5 & 56,2 & 4,5 & 0,4 & 3 & 7,9 \\
\hline Guelph $\left(43,7^{\circ} \mathrm{N}-200 \mathrm{~m}\right)$ & 20 & \multicolumn{2}{|c|}{46} & \multicolumn{2}{|c|}{34} & - & 7,1 \\
\hline
\end{tabular}


TABLEAU 2

Principales données culturales.

Cultural data.

\begin{tabular}{|c|c|c|c|c|c|c|c|}
\hline & & Variété & Date semis & $\begin{array}{c}\text { Densité } \\
\text { pieds } / \mathrm{m}^{2}\end{array}$ & $\begin{array}{c}\text { Floraison } \\
\text { femelle }\end{array}$ & Récolte & $\begin{array}{c}\text { Rendement } \\
\text { à } 15 \% \\
\text { d'humidité } \mathrm{qx} / \text { ha }\end{array}$ \\
\hline Colmar 1 & 1983 & $\uparrow$ & $29 / 04$ & $7,4^{*}$ & $18 / 07$ & $19 / 09$ & 83 \\
\hline Colmar 1 & 1984 & & $24 / 04$ & 8,5 & $30 / 07$ & $15 / 10$ & 92 \\
\hline Colmar 2 & 1983 & & $29 / 04$ & 7,7 & $18 / 07$ & $20 / 09$ & 100 \\
\hline Colmar 2 & 1984 & & $25 / 04$ & 8,3 & $8 / 08$ & $23 / 10$ & 78 \\
\hline Gundolsheim & 1983 & & $28 / 04$ & 6,2 & $20 / 07$ & $21 / 09$ & 66 \\
\hline Gundolsheim & 1984 & 1 & $24 / 04$ & 8,5 & $31 / 07$ & $17 / 10$ & 95 \\
\hline Flaxlanden & 1983 & DEA & $29 / 04$ & 8,0 & $22 / 07$ & $30 / 09$ & 103 \\
\hline Flaxlanden & 1984 & 1 & $20 / 04$ & 8,8 & $6 / 08$ & $24 / 10$ & 85 \\
\hline Altkirch 1 & 1983 & & $29 / 04$ & 9,5 & $25 / 07$ & $31 / 10$ & 80 \\
\hline Altkirch 1 & 1984 & & $23 / 04$ & 9,1 & $2 / 08$ & $22 / 10$ & 87 \\
\hline Altkirch 2 & 1983 & & $3 / 05$ & 7,8 & $25 / 07$ & $31 / 10$ & 86 \\
\hline Altkirch 2 & 1984 & & $29 / 04$ & 8,9 & $2 / 08$ & $24 / 10$ & 86 \\
\hline Mons & 1983 & $\downarrow$ & $6 / 05$ & 8,0 & $3 / 08$ & $13 / 10$ & 72 \\
\hline Guelph & 1984 & P 3857 & $\left.\begin{array}{l}17 / 05 \\
14 / 06 \\
29 / 06\end{array}\right\}$ & $7,5^{* *}$ & $\left.\begin{array}{r}2 / 08 \\
15 / 08 \\
29 / 08\end{array}\right\}$ & $10 / 10$ & $\begin{array}{l}71 \\
- \\
-\end{array}$ \\
\hline
\end{tabular}

* Densités observées à la levée.

** Densités obtenues après éclaircissage au stade 3 feuilles.

dolsheim 1983, Flaxlanden 1984, Colmar 1 1983, 1984), les caractéristiques mécaniques du profil étaient contraignantes pour l'enracinement.

Les principales données culturales sont résumées dans le tableau 2.

Les observations effectuées ont été celles décrites antérieurement (PICARD et al., 1985). Par rapport à celles-ci, seules les fréquences de mesures ont varié selon les sites. Sur les parcelles de Colmar 1 ainsi qu'à Mons et à Guelph, celle-ci était d'une fois par semaine. Sur toutes les autres, les prélèvements ont été effectués aux stades 6-8 feuilles, à la floraison femelle et à la récolte. On se proposait en effet, non pas de reprendre une étude détaillée du rythme d'émission des racines adventives, mais de vérifier qu'à certaines dates les nombres de racines sont, ou non, comparables et d'apprécier l'importance des écarts aux moyennes observées. Pour chaque plante ont été comptés : le nombre de feuilles visibles et étalées (ligule dégagée), le nombre d'entre-nœuds portant des racines adventives, et pour chaque entre-nœud le nombre de racines primaires allongées (longueur supérieure à $3 \mathrm{~cm}$ ).

Du fait de la diversité des équipements sur les lieux d'étude - domaines expérimentaux, parcelles chez des agriculteurs - les températures utilisées pour l'interprétation des résultats furent les températures air standard. La parcelle la plus éloignée d'un poste météo en était à $16 \mathrm{~km}$.

Les traitements statistiques des résultats ont porté sur des tests de comparaison de moyennes, des tests de $\mathrm{Chi}^{2}$ pour la comparaison des distributions, et, des recherches de régression linéaire entre les différents facteurs. Les tests de $\mathrm{Chi}^{2}$ (DAGNELIE, 1980) permettent de comparer les effectifs de plantes ayant un nombre donné de racines à un entre-nœud en fonction de ce nombre et du lieu d'observation et de conclure à la différence ou non des effectifs selon les lieux.

\section{RÉSULTATS ET DISCUSSION}

\section{A. Etude stationnelle}

\section{Distribution racinaire par entre-nœud ou groupe d'entre-nouds}

\section{a) Résultats}

L'étude précédente a permis de mettre en évidence 2 catégories d'entre-nœuds : E2 à E5, pour lesquels le nombre de racines par entre-nœud varie peu de plante à plante (PICARD et al., 1985) ; E6 à E8, pour lesquels le nombre de racines est apparu plus fluctuant.

Par conséquent, afin de réduire le nombre de comparaisons à effectuer, on se limitera à 2 entre-nœuds représentatifs, l'un de la première phase (E2), l'autre de la seconde phase (E6). Ces résultats seront complétés par l'étude des nombres cumulés de racines portées par les entre-nœuds 2 à 5 , et 6 à 8 .

+ Pour E2, le nombre moyen de racines par entrenœud varie de 3,2 à 3,9 avec un coefficient de variation (C.V.) de 13 à 23 p. 100 (fig. 1a, fig. 2). La dispersion intra-parcellaire, appréciée par les C.V. et les histogrammes de fréquences relatives (site par site), est faible, quels que soient les lieux d'observations. Une analyse de variance effectuée sur l'ensemble de la population montre que la variabilité inter-parcellaire est du même ordre de grandeur que la précédente, l'analyse de variance ne révélant pas de différence $(\mathrm{F}=0,45$ pour $12 \mathrm{dl}$ ), et que le nombre moyen de racines est très homogène. Il est toutefois possible de distinguer 3 classes de moyennes qui se chevauchent (tabl. 3). Par contre, des différences statistiquement significatives entre sites apparaissent dans la forme des distributions et sont mises en évidence par les calculs 


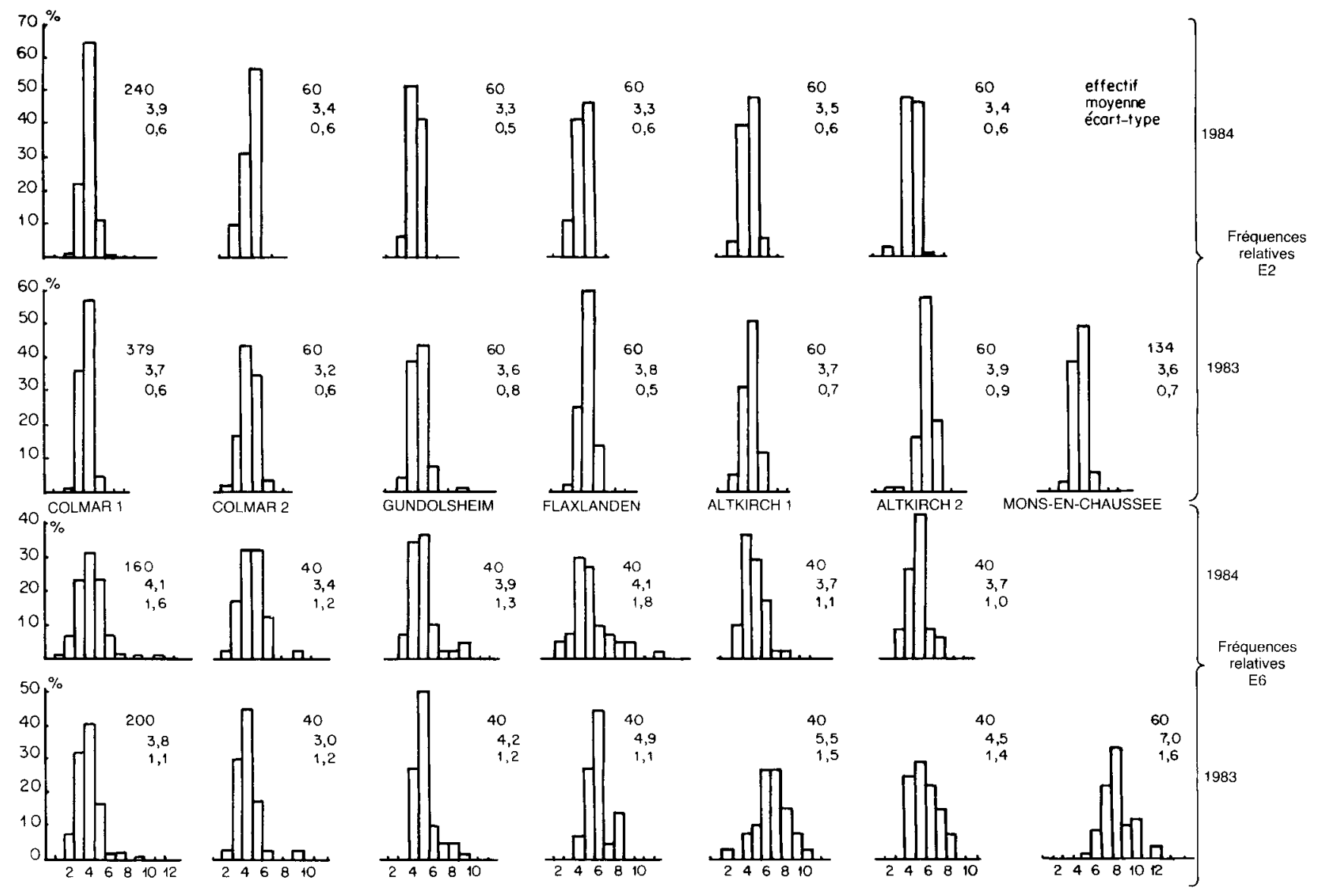

Figure 1.

Diagrammes de fréquence

Frequency graphs.

a) Distributions racinaires sur E2 et E6 en fonction des sites pour la

a) Root distribution for E2 and E6 relative to location (cv. DEA). variété $D E A$.

TABLEAU 3

Moyennes et résultats des comparaisons multiples de moyennes du nombre total de racines portées par entre-noud. Tests de NEWMANN \& KEUIS. Seuils $5 \%$.

Total root number per internode (means, multiple range test) relative to :

a) Etude stationnelle.

Locations.

\begin{tabular}{|c|c|c|c|c|c|}
\hline & & $\mathrm{E} 2$ & E2 à 5 & E6 & E6 à 8 \\
\hline Colmar 1 & 1983 & 3,6 bc & $12,3 \quad \mathrm{c}$ & 3,8 bc & 23,9 \\
\hline Colmar 1 & 1984 & $3,8 \quad \mathrm{c}$ & $11,6 \mathrm{abc}$ & $4,1 \quad b c$ & $15,6 \mathrm{a}$ \\
\hline Colmar 2 & 1983 & $3,2 \mathrm{a}$ & $11,7 \mathrm{abc}$ & $3,0 \mathrm{a}$ & 24,6 \\
\hline Colmar 2 & 1984 & $3,4 \mathrm{ab}$ & $11,6 \mathrm{abc}$ & $3,4 \mathrm{ab}$ & 23,8 \\
\hline Gundolsheim & 1983 & 3,6 bc & $10,9 \mathrm{a}$ & 4,2 bc & 23,4 \\
\hline Gundolsheim & 1984 & $3,3 \mathrm{ab}$ & $11,4 \mathrm{ab}$ & 3,9 bc & 23,8 \\
\hline Flaxlanden & 1983 & $3,8 \quad \mathrm{c}$ & $11,2 \mathrm{ab}$ & 4,9 de & 23,5 \\
\hline Flaxlanden & 1984 & $3,3 \mathrm{ab}$ & $11,8 \mathrm{abc}$ & 4,1 bc & 20,1 cde \\
\hline Altkirch 1 & 1983 & 3,7 bc & 13,8 de & $5,5 \quad \mathrm{e}$ & $17,1 \mathrm{abc}$ \\
\hline Altkirch 1 & 1984 & $3,5 \mathrm{abc}$ & $11,7 \mathrm{abc}$ & $3,7 \mathrm{bc}$ & $24,4 \quad f g$ \\
\hline Altkirch 2 & 1983 & $3,9 \mathrm{c}$ & $12,1 \quad b c$ & $4,5 \mathrm{~cd}$ & 19,1 bcd \\
\hline Altkirch 2 & 1984 & $3,4 \mathrm{ab}$ & $13,2 \quad \mathrm{~d}$ & 3,7 bc & 21,2 def \\
\hline Mons & 1983 & 3,6 bc & 13,9 & $7,0 \quad f$ & $15,9 \mathrm{ab}$ \\
\hline
\end{tabular}

b) Influence de la date de semis (Guelph).

Sowing dates.

\begin{tabular}{|c|c|c|c|c|c|c|}
\hline Date de semis & $\mathrm{E} 2$ & E6 & \multicolumn{2}{|c|}{ E2 à E5 } & \multicolumn{2}{|c|}{ E6 à E8 } \\
\hline 1 & $4,3 a$ & $8.5 \mathrm{~b}$ & 17,0 & $c$ & 38,2 & d \\
\hline 3 & $3,9 \mathrm{a}$ & 9,6 b & 16,2 & $c$ & 41,1 & de \\
\hline 2 & $4,5 \mathrm{a}$ & $9,8 \quad b$ & 16,7 & c & 42,4 & $\mathrm{C}$ \\
\hline
\end{tabular}



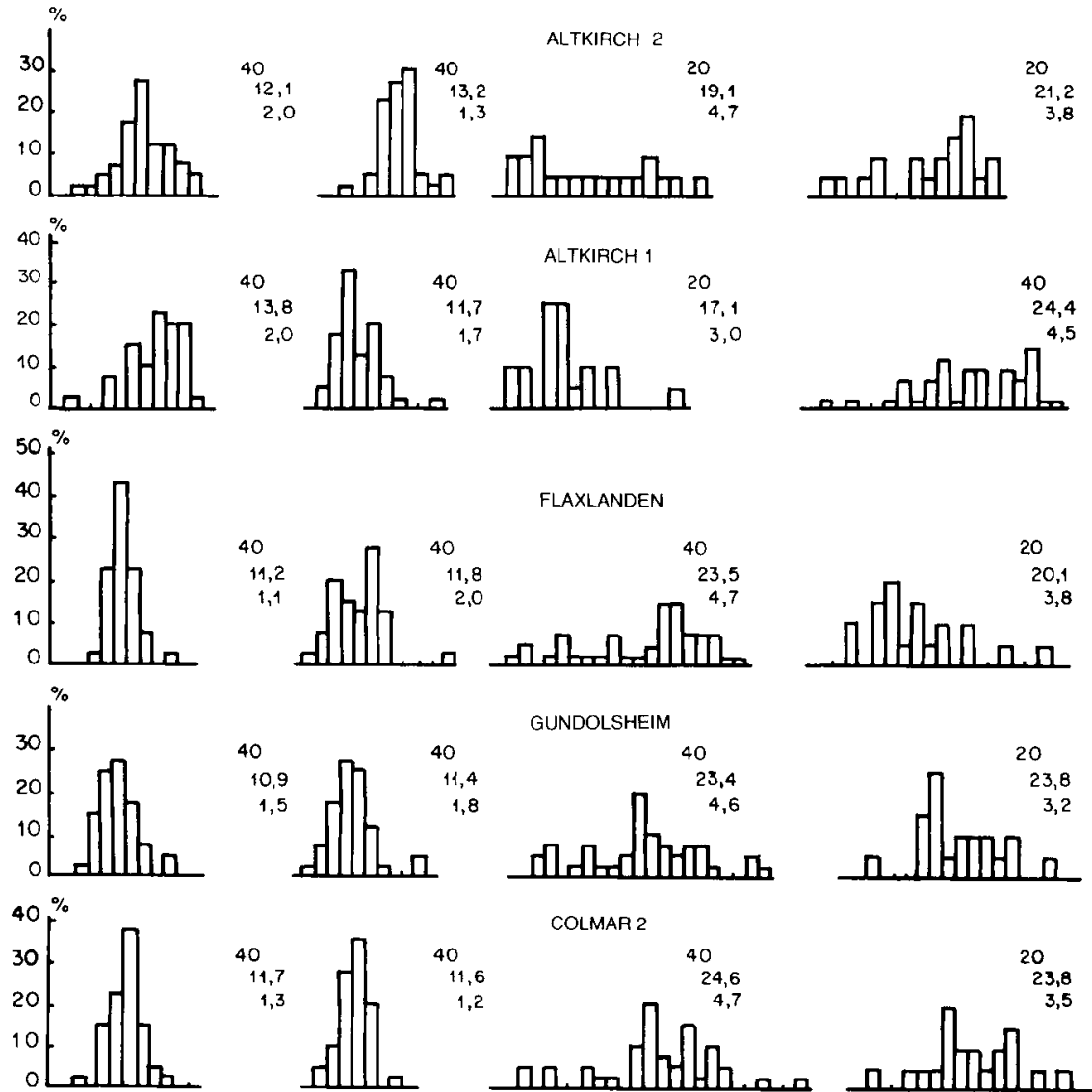

COLMAR 2
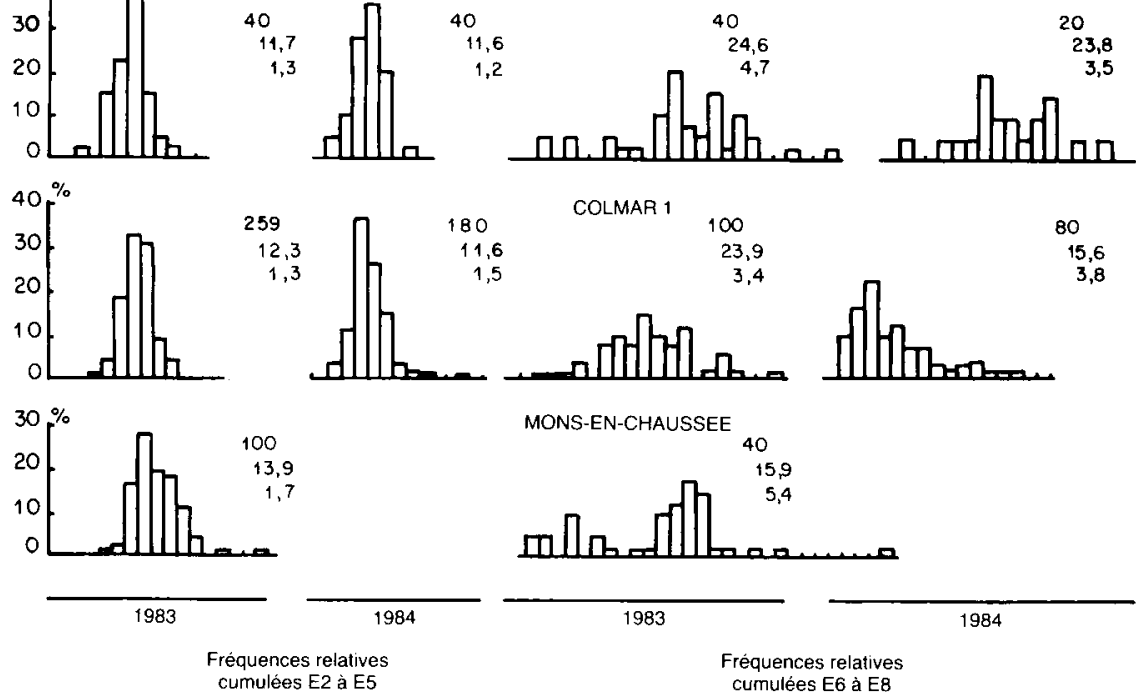

Figure 1

Diagrammes de fréquence.

b) Distributions racinaires sur E2 à 5 et 66 à 8 en fonction des sites pour la variété DEA.

de $\mathrm{Chi}^{2}$. Le tableau 4 montre un effet année non significatif pour E2. Les raisons de ces différences n'ont pas pu être explicitées.

+ Pour E2 à E5, les dispersions intra- et interparcellaires sont également faibles (fig. lb). Cependant, en ce qui concerne la variabilité inter-parcellaire, l'analyse de variance permet de différencier essentiellement 3 groupes de sites d'importance très inégale (tabl. 3a). Sur 10 d'entre eux, de 10,9 à 12,3 racines ont été dénombrées sur ces 4 entre-nœuds ; le second groupe comprend la placette Altkirch 2, 1984, où les plantes portent 13,2 racines; enfin, la placette Altkirch 1, 1983 (13,8 racines ; statistiquement, également non différente d'Altkirch 2,1984), peut être comparée à celle de Mons, où la moyenne est de 13,9 racines. Par ailleurs, un effet site et un effet année sur les formes
Frequency graphs.

b) Root distribution for E2 to 5 and $E 6108$ relative 10 location (c) $D E A$ ).

des distributions ont nettement été mis en évidence (tabl. 4).

+ Pour l'entre-nœud E6, la dispersion intraparcellaire est supérieure de 16 p. 100 à celle des entrenœuds inférieurs (fig. 1a ; fig. 2). La variabilité interparcellaire est significative pour cet entre-nœud $(\mathrm{F}=8,03$ pour 2 et $12 \mathrm{dl}$ ). Six sous-ensembles de moyennes sont individualisés (tabl. 3a). Pour les plantes issues du site de Colmar 2, 1983 et 1984, le nombre moyen de racines sur E6 est faible, bien que celui concernant la somme des entre-nœuds 6 à 8 pour le même site soit tout à fait comparable à son homologue pour les autres placettes étudiées. De plus, les pieds prélevés à Altkirch 1, 1983, Flaxlanden 1983 et à Mons portent significativement plus de racines sur E6 que le reste de la population. 

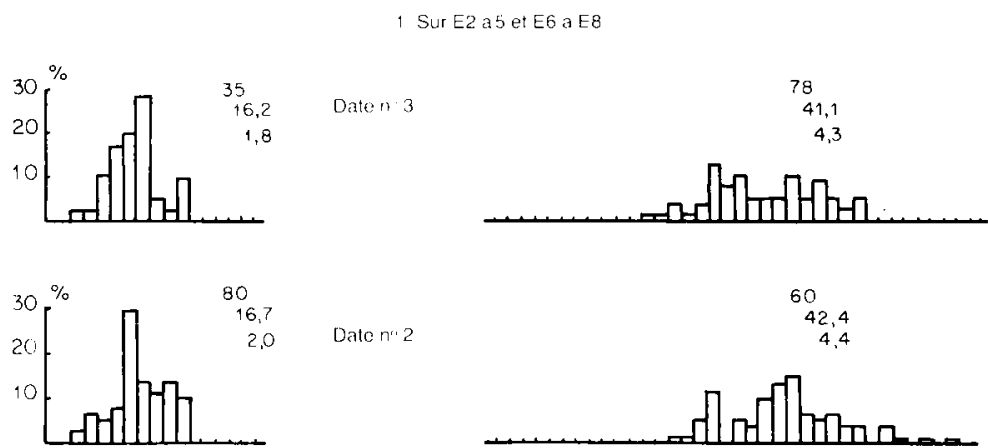

Date n'2 60
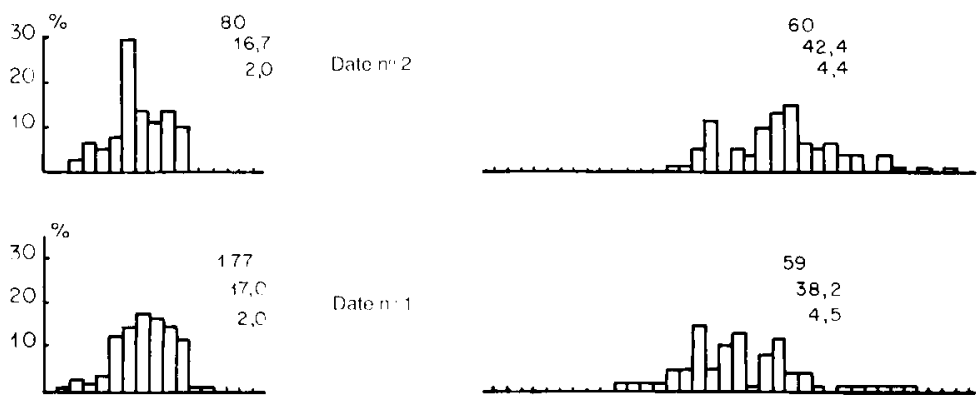

Daten' 38,2

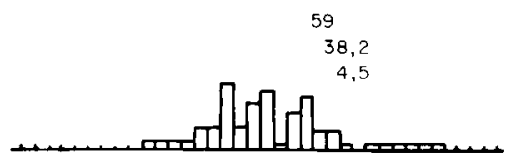

E. 6 j 8

2. Sur E2 et E6

Fréquences relatives : nombre de racines sur E2 et E6 sur $P 3851$ en fonction de la date de semis en 1984

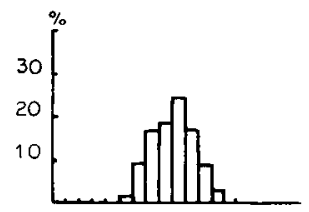

315

8,5
1,6
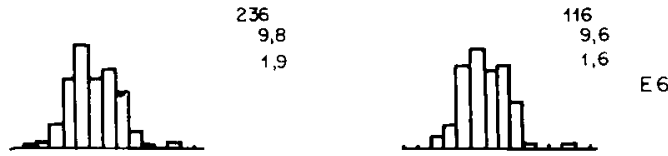

Date no 1

Date $n^{\circ} 2$

Date $n^{\circ} 3$

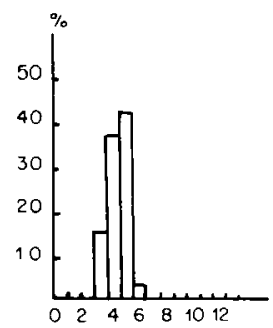

259

4,3
0,8

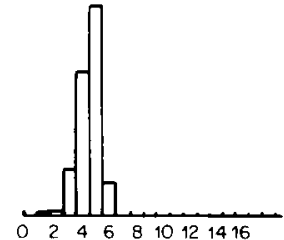

192

4,5
0,9

150

3,9

Figure 1

Diagrammes de fréquence.

$\begin{array}{ll}\text { c) Distributions racinaires (fréquences relatives) en fonction de la } & \text { c) Root distributior } \\ \text { date de semis pour la variété P } 3851 . & \text { date (cv. P 385I). }\end{array}$

Frequency graphs.

TABLEAU 4

Résultats des tests de Chi Carré

$\mathrm{Chi}^{2}$ test result.

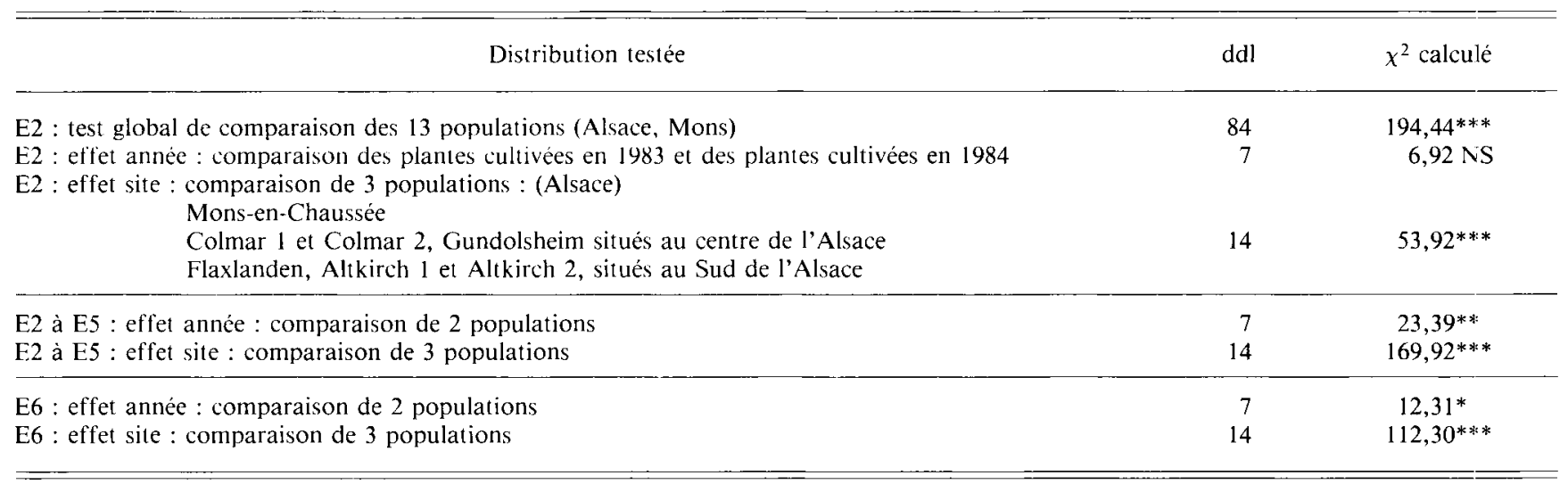




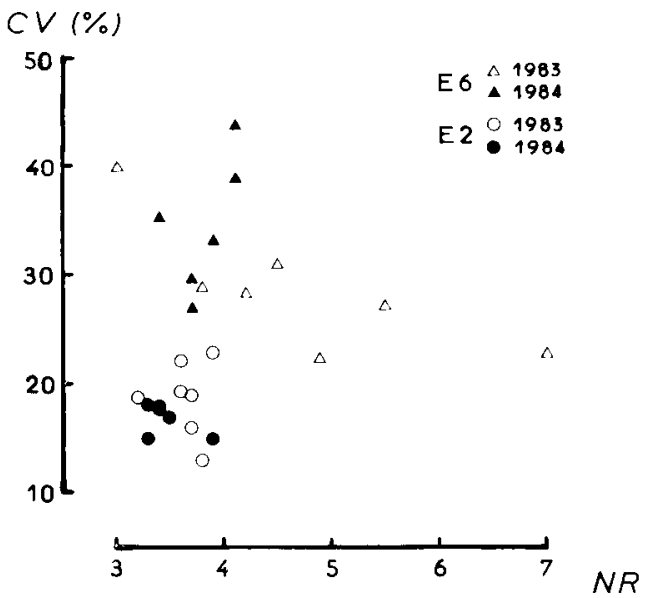

Figure 2

Relation entre le nombre moyen de racines (NR) par entre-noud et le coefficient de variation des observations (C.V.).

Relation between the mean number of roots (NR) per internode and the variation coefficient $(\mathrm{CV})$ of the observations.

+ En ce qui concerne l'ensemble des entre-nœuds E6 à E8, il y a une forte variabilité intra-parcellaire et les histogrammes de fréquences relatives sont très différents (fig. 1b). La dispersion inter-parcellaire des résultats est également très importante. Les différences observées sur les entre-nœuds 6 à 8 sont reliées en partie à l'importance de l'émission sur E8. A Colmar 1, 1984, Mons 1983 et Altkirch 1, 1983, il n'y a pas de racines sur E8. A Altkirch 2, 1983 et 1984 et Flaxlanden 1984, l'émission ne concerne qu'une partie des plantes, alors que, pour l'ensemble des autres sites, il y a émission suivie d'élongation pour tous les pieds.

\section{b) Discussion}

Sur l'ensemble des sites observés, les nombres de racines par entre-nœud varient d'abord en fonction du rang de l'entre-nœud. Pour les premiers d'entre eux (E2 à E5) les variabilités intra-parcellaires sont relativement plus faibles que pour les suivantes (E6 à E8). Les variabilités inter-parcellaires, initialement comparables aux précédentes (cas de E2), augmentent avec le rang des entre-nœuds puisqu'il devient possible de distinguer certaines parcelles à partir du nombre total de racines pour les entre-nœuds E2 à E5. Les données disponibles, notamment les données climatiques et les états des profils à la floraison, ne permettent pas de rendre compte des écarts observés sauf, éventuellement, dans le cas de Mons qui se situe à la limite Nord pour la culture de « Dea », ce qui pourrait expliquer le faible nombre de racines sur les derniers entre-nœuds.

Toujours en ce qui concerne la variabilité interparcellaire, à partir de E6 et E6 à E8, les différences observées entre sites sont en grande partie imputables à ce qui se passe à l'entre-noud 8. Dans le cas du maïs, les conditions de milieu agissent non seulement sur le nombre de racines portées par E6 et E7 mais peuvent empêcher ou favoriser l'émission sur E8. Chez les graminées qui tallent (blé, certaines variétés de riz), le nombre de racines par talle apparaît davantage prédéterminé et la régulation due au milieu porte plutôt sur le nombre de talles fertiles (PINTHUS, 1969 ; HARADA et al., 1984a et $b$; YAMAZAKI \& HARADA, 1984).
L'absence d'émission suivie d'élongation en E8 pose un problème car elle semble sans conséquence pour le rendement en grains de la culture.

Deux hypothèses peuvent être émises pour expliquer cet état de fait : la première consisterait à dire que la présence d'un nombre important de racines à fort diamètre inhibe l'activité de celles ayant un diamètre faible, notamment du système séminal, bien que ce dernier puisse rester actif durant toute la durée du cycle (KoZINKA, 1977).

D'autre part, les racines issues de l'entre-nœud 8, et dans une certaine mesure de l'entre-nœud 7, pourraient servir d'organes de stockage, comme semble le montrer la variabilité des poids secs (baisse suivie d'une augmentation) entre la floraison et la récolte de la partie du système racinaire localisée dans les 10 premiers $\mathrm{cm}$ sous le pied de maïs (KLOCKER, 1984). Cette variation de poids sec des racines pourrait être imputable au changement de la masse volumique de ces grosses racines. Ce dernier point mérite d'être élucidé.

Enfin, au vu de la dispersion du nombre de racines sur les différents entre-nœuds, nous avons cherché à savoir si une partie de la variabilité observée ne pourrait être expliquée par des phénomènes de compensation et d'autorégulation de l'émission et de l'élongation racinaire entre les différents entre-nœuds. Pour cela, nous avons calculé systématiquement les corrélations entre nombre de racines à un entre-nœud donné et nombre de racines à l'entre-nœud suivant. Les relations obtenues n'expliquent que 8 à 17 p. 100 de la variabilité observée. Les phénomènes de compensation sont donc pratiquement inexistants.

\section{Relations entre le nombre total de racines et le nombre total de feuilles}

\section{a) Résultats}

Le tableau 5 donne, pour chacun des sites, le nombre moyen de racines primaires allongées à la fin de l'émission en fonction du nombre total de feuilles. Si, en moyenne, le nombre total de racines primaires a tendance à augmenter avec le nombre de feuilles, les différences ne sont pas statistiquement significatives. Par ailleurs, cette tendance ne se vérifie pas systématiquement sur chacune des placettes ; ainsi, il n'y a que pour Altkirch 1, 1983, que le nombre de racines des plantes de 16 feuilles est statistiquement supérieur à celui des plantes de 14 et 15 feuilles. De plus, il n'apparaît aucune relation entre le nombre final de feuilles de l'ensemble des plantes prélevées prises individuellement, et le nombre de racines primaires sur les entrenœuds 2 à 5 , sur les entre-nœuds 6 et 7 et enfin sur l'entre-nœud 8.

\section{b) Discussion}

Le dispositif expérimental utilisé ne permet pas de mettre en évidence, au stade floraison, une relation directe entre nombre total de racines émises et nombre total de feuilles pour une plante de mais. Mais ceci est vraisemblablement dû à la faible gamme de variation observée.

En effet, chez certaines variétés de riz (YAMAZAKI et $a l ., 1981$; MORITA et $a l ., 1983 a$ et $b$ ) le diamètre d'un 
TABLEAU 5

Nombre total de racines en fonction du nombre de feuilles. Total root number in relation to of leaf number.

\begin{tabular}{|c|c|c|c|c|c|c|c|c|c|c|c|c|c|}
\hline Nombre de feuilles & & \multicolumn{3}{|c|}{14} & \multicolumn{3}{|c|}{15} & \multicolumn{3}{|c|}{16} & \multicolumn{3}{|c|}{17} \\
\hline Mons-en-Chaussée & 1983 & 3 & 25,0 & $(5,19)$ & 23 & 29,9 & $(5,79)$ & 14 & 30,4 & $(4,07)$ & & - & \\
\hline Colmar 1 & 1983 & 3 & 30,7 & $(2,08)$ & 18 & 35,5 & $(4,47)$ & 66 & 36,1 & $(3,72)$ & 12 & 37,3 & $(4,33)$ \\
\hline Colmar 2 & 1983 & & - & & 6 & 35,0 & $(6,60)$ & 29 & 37,9 & $(4,29)$ & 4 & 36,5 & $(2,38)$ \\
\hline Gundolsheim & 1983 & 7 & 38,6 & $(7,16)$ & 19 & 35,0 & $(3,82)$ & 10 & 36,6 & $(3,65)$ & & - & \\
\hline Flaxlanden & 1983 & & - & & 17 & 36,5 & $(4,10)$ & 18 & 37,6 & $(3,72)$ & 3 & 37,0 & $(1,73)$ \\
\hline Altkirch 1 & 1983 & 6 & 30,2 & $(2,78)$ & 25 & 30,7 & $(3,60)$ & 9 & 34,7 & $(5,27)$ & & 一 & \\
\hline Altkirch 2 & 1983 & 8 & 28,5 & $(6,21)$ & 26 & 28,8 & $(5,74)$ & 6 & 27,5 & $(2,66)$ & & - & \\
\hline Colmar 1 & 1984 & & 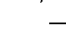 & & 10 & 27,7 & $(4,84)$ & 64 & 27,5 & $(3,79)$ & 5 & 29,4 & $(4,15)$ \\
\hline Colmar 2 & 1984 & & - & & 16 & 33,3 & $(4,76)$ & 18 & 33,5 & $(5,56)$ & 4 & 35,6 & $(5,31)$ \\
\hline Gundolsheim & 1984 & 8 & 34,3 & $(8,10)$ & 19 & 34,2 & $(4,42)$ & 9 & 36,5 & $(4,97)$ & & - & \\
\hline Flaxlanden & 1984 & 9 & 29,7 & $(4,84)$ & 22 & 33,8 & $(5,83)$ & 9 & 32,9 & $(2,31)$ & & - & \\
\hline Altkirch 1 & 1984 & & - & & 3 & 38,0 & $(7,21)$ & 18 & 34,7 & $(8,91)$ & 17 & 35,5 & $(3,59)$ \\
\hline Altkirch 2 & 1984 & & - & & 10 & 30,8 & $(7,13)$ & 29 & 30,8 & $(6,78)$ & & - & \\
\hline \multicolumn{2}{|l|}{ Moyenne } & \multicolumn{3}{|c|}{31,5} & \multicolumn{3}{|c|}{32,5} & \multicolumn{3}{|c|}{32,8} & \multicolumn{3}{|c|}{35,5} \\
\hline
\end{tabular}

$3 \quad$ Nombre de plantes prélevées

25,0 Nombre moyen de racines.

$(5,19)$ Ecart-type.

entre-nœud semble déterminer le nombre de racines primaires et leur diamètre. Or le diamètre d'un entrenœud est en général en liaison avec la vigueur de la plante. Il reste à vérifier si une relation de ce type existe chez le maïs et s'il est possible de relier l'émission des racines primaires à la taille de l'entre-nœud porteur. Si cela était le cas, une différence de vigueur entre les plantes issues des divers sites se traduirait par une réduction ou une augmentation de l'émission racinaire et permettrait d'expliquer une partie des différences observées.

\section{B. Influence de la date de semis}

\section{Résultats}

Les semis échelonnés sur 6 semaines (essais de Guelph) induisent un décalage des cycles de culture et des floraisons réparties sur tout le mois d'août (tabl. 2). Pour un même entre-nœud, le nombre de racines par entre-nœud, ainsi que sa variabilité paraissent indépendants de la date de semis (tabl. 3b). Les nombres de racines varient avec les entre-nœuds mais la variabilité, exprimée par les coefficients de variation, est la même pour E6 et E6 à E8 que pour E2 et E2 à $\mathrm{E} 5$ (fig. 1c).

Le nombre total des racines allongées croît avec la somme des températures selon une relation doublement logarithmique (fig. 3). Le nombre moyen d'entre-nouds portant des racines allongées, lui, augmente linéairement avec le nombre de feuilles visibles (fig. 4).

Des tests de comparaison des coefficients de régression montrent que dans les 2 cas, les courbes obtenues pour chacune des dates de semis peuvent être confondues. Par conséquent, l'échelonnement des semis ne modifie pas la cinétique de mise en place de l'appareil racinaire. En particulier, le synchronisme existant entre le phyllochrone (GOUNOT et al., 1980) et l'émis-

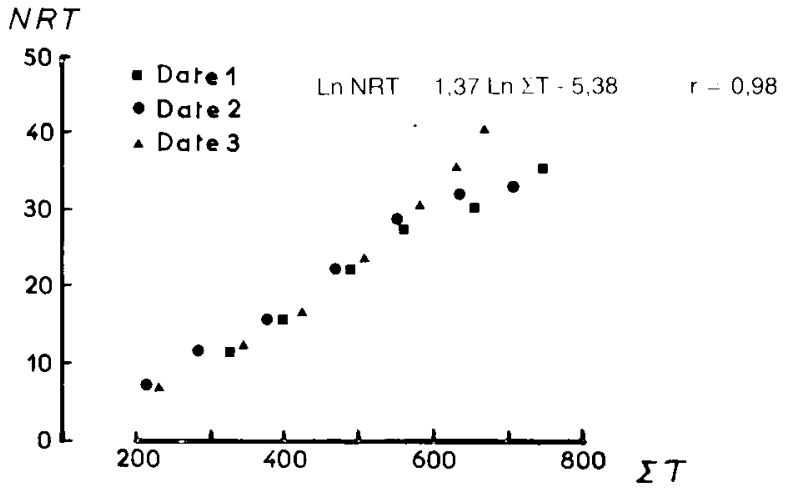

Figure 3

Nombre total de racines allongées (NRT) en fonction de la somme des températures $(T)$ supérieures à $6{ }^{\circ} \mathrm{C}$.

Primary root emission in relation to of heat sum units.

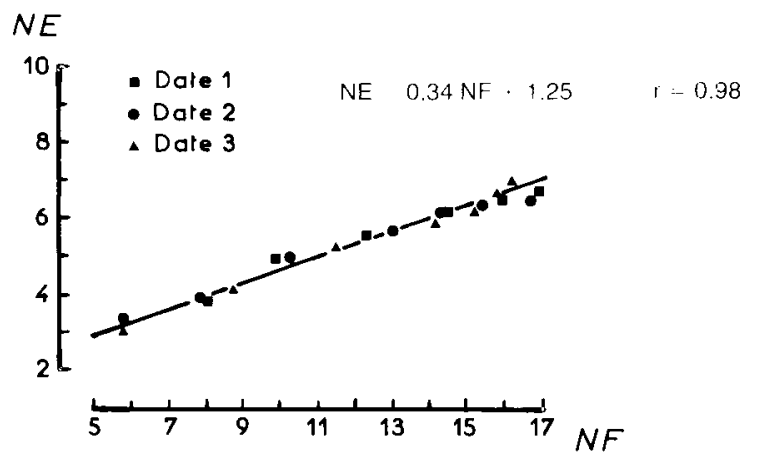

Figure 4

Nombre moyen d'entre-nouds (NE) portant des racines allongées en fonction du nombre de feuilles visibles (NF).

Root emission per internode in relation to of phyllochron.

sion des racines primaires n'est pas affecté par le décalage des dates de semis. 


\section{Discussion}

Le fait de décaler les dates de semis ne modifie pas les évolutions déjà constatées sur les nombres moyens de racines par entre-nœud. Le synchronisme entre l'émission des racines et celui des feuilles (PICARD $e t$ $a l ., 1985)$ se retrouve également : les coefficients des droites de régression ne sont pas identiques, mais les différences observées peuvent être dues au fait que les variétés en essai n'étaient pas les mêmes. Ce type de synchronisme a d'ailleurs été également observé pour certaines variétés de riz par KAWATA \& SOEJIMA (1974) et YAMAZAKI \& HARADA $(1982,1984)$. Par contre, ces derniers notent un écart constant de 3 entrenœuds entre l'apparition des feuilles et celle des racines primaires, alors que, pour le maïs, cet écart augmente lui même linéairement avec le nombre de feuilles. Dans le premier cas, l'âge des entre-nœuds au moment de l'émission des racines primaires est sensiblement le même quelle que soit leur position sur la tige. Chez le maïs, par contre, les entre-nœuds sont d'autant plus âgés au moment de l'émission que leur rang est élevé.

\section{CONCLUSION ET PERSPECTIVES}

Après une étude détaillée, en un lieu, du rythme d'apparition des racines de maïs et en l'absence de références bibliographiques, l'étude entreprise a eu pour objectif d'évaluer l'importance de la variabilité du nombre de racines primaires émises en fonction des conditions pédo-climatiques.
Les résultats obtenus antérieurement ne sont pas remis en cause : le nombre de racines primaires par entre-nœud qui est fonction du rang de celui-ci, diminue de l'entre-nœud 2 à l'entre-nœud 4 puis réaugmente au-delà. Dans les conditions de la grande culture et en sol de limon, la variabilité inter-parcellaire est de l'ordre de grandeur de la variabilité inter-parcellaire pour les premiers entre-nœuds. Elle augmente cependant plus vite que cette dernière avec le rang des entrenœuds et est la plus élevée aux entre-nœuds 8 et 9 . A ces niveaux, l'émission et l'élongation des racines semblent directement liées aux états de croissance à la floraison. Il n'apparaît pas de relation étroite entre le nombre de racines primaires à un entre-nœud et celui aux autres entre-nœuds. Un décalage même important de la date de semis ne modifie ni la chronologie d'émission des racines par rapport à celle des feuilles, ni le nombre de racines primaires par entre-nœud.

Il faut désormais élucider les raisons des variabilités observées, en analyser les conséquences sur la nutrition hydrique et minérale des plantes et le rendement du maïs. Nos connaissances sur ce sujet sont en effet très limitées. Mis à part les travaux de TARDIEU \& MANICHON (1986a et $b$ ), qui ont réussi, par une méthode de cartographie racinaire à relier enracinement et structure du sol, les autres résultats disponibles (cf. la revue par BONHOMME, 1983) concernant l'évolution de la masse ou de la longueur du système racinaire (ALLMARAS \& NELSON, 1971; MENGEL \& BARbER, 1974) n'intègrent pas suffisamment l'état du profil.

Reçu le 25 avril 1986 Accepté le 15 septembre 1987.

\section{RÉFÉRENCES BIBLIOGRAPHIQUES}

Allmaras R. R., Nelson W. W., 1971. Corn (Zea mays L.) root configuration as influenced by some row-interrow variants of tillage and straw mulch management. Proc. Soil Sci. Soc. Am., 35, 974-980.

Bonhomme R., 1983. Mise en place des appareils foliaire et racinaire chez le mais. Coll. Physiol. mais, Royan, 15-17/03/1983. I.N.R.A. Paris, 1984, $574 \mathrm{p}$.

Dagnelie P., 1980. Théorie et méthodes statistiques. Gembloux, Presses Agronomiques (vol. 2), $463 \mathrm{p}$.

Duburcq J. B., Bonhomme R., Derieux M., 1983. Durée des phases végétatives et reproductrices chez le maïs. Influence du génotype et du milieu. Agronomie, 3 (10), 941-946.

Gounot M., Atry M., N'Kandza J., Yu O., 1980. Photosynthèse nette et rythme d'apparition des feuilles chez le dactyle (Dactylis glomerata L.). C. R. Acad. Sci. Ser. D, Sci. nat., 290, 1257-1260.

Harada J., Yamazaki K., Nakamoto T., Miyake A., Umeda T., 1984a. The relationship between the number of primary roots and yield components in rice plants grown in farmer paddy fields. I. Analyses per hill and per plant. Jpn J. Crop Sci., 53 (3), 307-312.

Harada J., Yamazaki K., Umeda T., Miyake A., Nakamoto T., $1984 b$. The relationship between the number of primary roots and yield components in rice plants grown in farmer paddy fields. II. Analyses per culm and per shoot unit. Jpn J. Crop Sci., 53 (3), 313339.

Kawata S., Soejima M., 1974. On superficial root formation in rice plants. Proc. Crop Sci. Soc. Jpn, 43 (3), 354-374.

Klocker M., 1984. Contribution à l'étude de la colonisation du sol par les racines en relation avec le développement du système aérien chez le mais. DEA-INPL-ENSAIA Nancy, $65 \mathrm{p}$.

Kozinka V., 1977. Conditions for "internal aeration » in the seminal root system of Zea mays L. Biologia, Bratislava, 34, 523-529.
Mengel D. B., Barber A., 1974. Development and distribution of the corn root system under field conditions. Agron. J., 66, 341-344.

Morita S., Yamazaki K., Kawata S., 1983a. Relationships between the growth directions of primary roots and their anatomical characters in rice plants. Jpn J. Crop. Sci., 52 (4).

Morita S., Yamazaki K., Kawata S., 1983b. Relationships between the growth directions of primary roots and their conductive capacities in rice plants. Jpn J. Crop. Sci., 52 (4).

Picard D., Jordan M.-O., Trendel R., 1985. Rythme d'apparition des racines primaires du maïs. I. Etude détaillée pour une variété en un lieu donné. Agronomie, 5 (8), 667-676.

Pinthus M. J., 1969. Tillering and coronal root formation in some common and durum wheat varieties. Crop Sci., 9, 267-272.

Russell W. K., Stuber C. W., 1983. Effects of photoperiod and temperature on the duration of vegetative growth in maize. Crop Sci., 23 (5), 847-850.

Russelle M. P., Wilhelm W. W., Olson R. A., Power J. F., 1984. Growth analysis based on degree days. Crop Sci., 24 (1), 28-32.

Tardieu F., Manichon H., 1986a. Caractérisation en tant que capteur d'eau de l'enracinement du maïs en parcelle cultivée. 1. Discussion des critères d'études. Agronomie, 6 (4), 345-354.

Tardieu F., Manichon H., 1986 $b$. Caractérisation en tant que capteur d'eau de l'enracinement du maïs en parcelle cultivée. II. Une méthode d'étude de la répartition verticale et horizontale des racines. Agronomie, 6 (5), 415-426.

Tollenaar M., Daynard T. B., Hunter R. B., 1979. Effect of temperature on rate of leaf appearence and flowering date for maize. Crop Sci., 19, 363-366. 
Tollenaar M., Hunter R. B., 1983. A photoperiod and temperature sensitive period for leaf number in maize. Crop Sci., 23 (3), 457-460.

Warrington I. J., Kanemasu E. T., 1983a. Corn growth response to temperature and photoperiod. I. Seedling - Emergence - Tassel initiation and anthesis. Agron. J., 75 (5), 749-754.

Warrington I. J., Kanemasu E. T., 1983b. Corn growth response to temperature and photoperiod. II. Leaf initiation and leaf appearence rates. Agron. J., 75 (5), 755-761.

Warrington I. J., Kanemasu E. T., 1983c. Corn growth response to temperature and photoperiod. III. Leaf number. Agron. J., 75 (5), 762-767.
Yamazaki K., Morita S., Kawata S., 1981. Correlations between the growth angles of crown roots and their diameters in rice plants. Jpn J. Crop. Sci., 50 (4), 452-456.

Yamazaki K., Harada J., 1982. The root system formation and its possible bearing on grain yield in rice plant. Jpn J. Crop Sci., 15 (3), 153-160.

Yamazaki K., Harada J., 1984. The relationship between the number of primary roots and yield components in rice plants grown in farmer paddy fields. III. Variation between paddy fields. Jpn J. Crop Sci., 53 (3), 320-325. 